\title{
ISÓTOPOS ESTABLES Y DIETA DE LOS CAZADORES RECOLECTORES DEL SUDOESTE BONAERENSE (ARGENTINA)
}

\author{
STABLE ISOTOPES AND DIET OF HUNTERS GATHERERS OF SOUTHWEST \\ OF BUENOS AIRES PROVINCE (ARGENTINA)
}

\author{
Clara Scabuzzo ${ }^{1}$, Romina Frontini ${ }^{2}$, Rodrigo Vecchi y Cristina Bayón ${ }^{3}$
}

\begin{abstract}
Se presentan los primeros resultados del análisis de isótopos estables $\left(\delta^{13} \mathrm{C}\right.$ y $\left.\delta^{15} \mathrm{~N}\right)$ obtenidos sobre restos humanos provenientes de sitios arqueológicos del sudoeste de la provincia de Buenos Aires (Argentina), datados en el Holoceno Tardío. Conjuntamente se informan los primeros datos de $\delta^{13} \mathrm{Ccol}$. sobre los distintos recursos faunísticos del sector.

El área de estudio se compone de diversos ambientes, destacándose los entornos acuáticos como la costa marina, los valles fluviales, las lagunas y el humedal. Los valores isotópicos de los recursos del sector indican que los animales marinos presentan los valores más enriquecidos en $\delta^{13} \mathrm{C}$; mientras que aquellas especies terrestres (coipo, ñandú y herbívoros grandes) presentan los valores más empobrecidos, que son indistinguibles entre ellos. Por su parte, las aves acuáticas y el peludo tienen valores intermedios. El estudio isotópico sobre restos humanos reflejó que la dieta de los individuos fue cárnica, que las proteínas de animales terrestres fueron predominantes y que en menor proporción se usaron recursos marinos. El registro arqueofaunístico del área resulta concordante con esta información. Finalmente, estos son los primeros resultados isotópicos para el sector, por lo que constituye un aporte preliminar que deberá continuar con la realización de nuevos estudios.

Palabras claves: cazadores-recolectores, isótopos estables, registro bioarqueológico y arqueofaunístico, región pampeana, Holoceno Tardío.

Results of the first stable isotopes analysis $\left(\delta^{13} \mathrm{C}\right.$ and $\left.\delta^{15} \mathrm{~N}\right)$ of archaeological sites located in the southwest of the province of Buenos Aires (Argentina) are presented. Both the construction of an isotopic ecology and prehistoric human diets of the sector are explored. The isotopic ecology indicates diverse environments in which marine animals have enriched $\delta^{13}$ Ccol. values; while terrestrial species such as coypo, greater rhea and large-sized herbivores have the poorest values that are indistinguishable among the different taxa. Furthermore, ducks and a hairy armadillo showed intermediate values. Human isotopic values indicate a diet based on the consumption of meat. These proteins came predominantly from terrestrial animals complemented by marine resources. The local archaeological record is consistent with this information, as the main faunal resources are terrestrial species; nevertheless a scarce representation of marine fauna, mainly Otariids, is registered. In this paper we present the first isotopic values for the studied area and thus it is a preliminary contribution that must be further investigated with new samples.
\end{abstract}

Key words: Hunter-gatherers, stable isotopes, human and faunal record, Pampean region, Late Holocene.

En las últimas décadas se utilizaron múltiples indicadores para acceder a la alimentación de las poblaciones prehistóricas. Entre otros se destacan los análisis de fitolitos, de ácidos grasos, de desgaste dental y de arqueofaunas. Sin embargo, aunque estos informan sobre el tipo de alimento consumido, solo posibilitan acceder indirectamente al rol de cada uno de los productos en la dieta. En los últimos años los análisis de isótopos estables permitieron efectuar una aproximación más precisa a la alimentación y conocer la importancia relativa de algunos alimentos (Ambrose 1993; Ambrose y Norr 1993; Barberena 2014; Froehle et al. 2012).

En la región pampeana argentina la subsistencia fue abordada predominantemente a partir de las asociaciones faunísticas y complementada con inferencias basadas en artefactos de molienda, fitolitos, residuos en alfarería y en instrumentos

1 CONICET-División Arqueología, Facultad de Ciencias Naturales y Museo, Universidad Nacional de La Plata, Unidad de Investigación Anexo Museo, Av. 60 y 122, La Plata, Argentina. clarascabuzzo@ hotmail.com

2 CONICET-Departamento de Humanidades, Universidad Nacional del Sur, 12 de Octubre y San Juan, Bahía Blanca, Argentina. frontiniromina@gmail.com; druyer79@hotmail.com

3 Departamento de Humanidades, Universidad Nacional del Sur, 12 de Octubre y San Juan, Bahía Blanca, Argentina. crisbayon@gmail.com 
líticos (Frére et al. 2010; Matarrese 2015; Mazzia y Flegenheimer 2015). Recientemente los análisis isotópicos tuvieron un impacto notable que se refleja en el creciente número de proyectos que incluyen esta línea de evidencia (Barrientos et al. 2015; Berón et al. 2009; Bonomo et al. 2013; Flegenheimer et al. 2002; Loponte y Acosta 2007; Martínez et al. 2009, 2012; Mendonça et al. 2013; Murgo y Aldazabal 2007; Politis et al. 2009; Scabuzzo y González 2007). Estos análisis permitieron ampliar el conocimiento concerniente a la explotación de recursos de los diversos ambientes y complementaron los estudios arqueofaunísticos convencionales. Sin embargo, su aplicación no fue homogénea en las distintas áreas de la región, ya sea por la conservación diferencial de los materiales o por la historia de las investigaciones.

En este trabajo se presentan los primeros resultados de esta línea de investigación aplicada a contextos del Holoceno Tardío en un sector del sudoeste bonaerense. Simultáneamente se informan cuatro nuevos fechados realizados sobre restos humanos. Aunque los resultados no son numerosos permiten discutir y analizar de manera preliminar la información paleodietaria sobre la base de estudios de isótopos estables $\left(\delta^{13} \mathrm{C}\right.$ y $\left.\delta^{15} \mathrm{~N}\right)$ realizados en restos humanos y aportar los primeros datos isotópicos acerca de los recursos faunísticos del área. Los análisis aquí presentados contribuirán a discutir el rol que tuvieron las diversas especies animales, incluyendo el aporte de la fauna pequeña en la dieta, cuyo consumo está evidenciado por el registro arqueofaunístico. Finalmente se discuten las similitudes y diferencias en una escala espacial amplia al comparar los datos con la información isotópica disponible para la región.

\section{Ambiente y Paleoambiente}

El sector en estudio está dentro del sudoeste bonaerense, en la llanura interpuesta entre las Sierras Australes y el mar (Figura 1A). Es una zona ecotonal con clima ventoso, lluvias entre 500 y $600 \mathrm{~mm}$ anuales y sequías frecuentes (Bróndolo y Zinger 1988). Fitogeográficamente se conforma una amplia zona transicional en la intersección del Distrito Austral de la Provincia Pampeana con el Distrito del Caldén de la Provincia del Espinal, con dominancia de vegetales del tipo C3 (Cabrera 1976). Zoogeográficamente, forma un ecotono, el Distrito Central sudoriental, que está interpuesto entre los Dominios Pampásicos de la subregión
Guayano-brasileña y Patagónico de la subregión Andino-patagónica (Ringuelet 1961).Varios cursos de agua, que nacen en las Sierras Australes y desembocan en el Océano Atlántico, atraviesan la llanura, entre ellos el río Sauce Grande y el arroyo Napostá Grande. Hacia el sur el litoral marítimo bordea todo el sector y hacia el continente se desarrolla la Barrera Medanosa Austral de varios kilómetros de extensión (Isla et al. 2001). Los estudios paleoambientales muestran cambios a lo largo del Holoceno. Para el Holoceno Tardío se describen condiciones de aridez y semiaridez; ca. 1.600 años a.p. se infiere un mejoramiento en el clima similar a las condiciones actuales (Quattrocchio et al. 2008).

\section{Antecedentes del Sector de Estudio}

Las investigaciones arqueológicas en el sector comenzaron en la década de 1960 con los trabajos de A. Austral y M.E. Pérez Amat, quienes realizaron excavaciones y recolecciones superficiales (Austral 1968; Pérez Amat y Scheines de Tiverovsky 1978). En las últimas décadas se excavaron nuevos sitios y se reestudiaron dichas colecciones incluyendo análisis tecnológicos, bioarqueológicos y arqueofaunísticos (Bayón et al. 2010; Frontini 2013; Scabuzzo 2013; Vecchi et al. 2013).

Las ocupaciones en el sector tienen edades desde 7.900 hasta 700 años a.p., aunque en este trabajo solo se considerarán los sitios del Holoceno Tardío. En este lapso los emplazamientos están entre 5 y $41 \mathrm{~km}$ de la costa y no hay registro de ocupaciones costeras, a diferencia de lo que ocurre en el Holoceno Temprano y Medio. Los sitios analizados son: Paso Mayor Y1S1-2 Niveles Superiores (PM NS), Paso Vanoli (PV), Villa Sapito (VS), García del Río II (GR II), Laguna Toro (LT), Laguna del Sauce (LS) y Puente de Fierro (PF) (Frontini 2013; Frontini y Bayón 2015a; Tomassini et al. 2014; Vecchi et al. 2013) (Figura 1B).

En relación con la explotación de fauna, las especies continentales y de gran porte, especialmente el guanaco, fueron preferencialmente utilizadas en Puente de Fierro, Paso Vanoli y Paso Mayor, representando ca. 27\% del NISP total (Frontini 2013; Frontini y Bayón 2015b). En Puente de Fierro el guanaco está seguido por el venado de las pampas $(\mathrm{NISP} \%=4,16)$ y hay un registro diverso de especies de porte menor que incluyen coipo (NISP\%=17,50), peludo (NISP $\%=2,2)$, pato (NISP $\%=2,33$ ), gallareta $(\mathrm{NISP} \%=1,84)$, macá $(\mathrm{NISP} \%=0,37)$ y chajá 
$($ NISP\%= 0,37) (Frontini y Bayón 2015a); se destaca además la presencia de restos de otáridos (NISP\%= 2,44). En Paso Mayor también se registran venado de las pampas (NISP $\%=2,5)$ y peludos $(\mathrm{NISP} \%=$ $5,5)$ como especies secundarias.

El registro bioarqueológico del sector da cuenta de la existencia de siete sitios con restos humanos; de estos seis poseen fechados radiocarbónicos que ubican los eventos de inhumación entre ca. 7.800 y 700 años a.p. Las prácticas mortuorias incluyen entierros primarios y secundarios, siendo más frecuentes los primarios; se inhumaron tanto adultos como subadultos de ambos sexos (Scabuzzo 2013). El número mínimo de individuos por sitio varía desde lugares donde se halló un único esqueleto hasta sitios donde se recuperaron más de siete individuos.

\section{Materiales y Métodos}

Los valores isotópicos de los alimentos se transfieren a los consumidores y cambian según diferentes variables como la vía fotosintética de

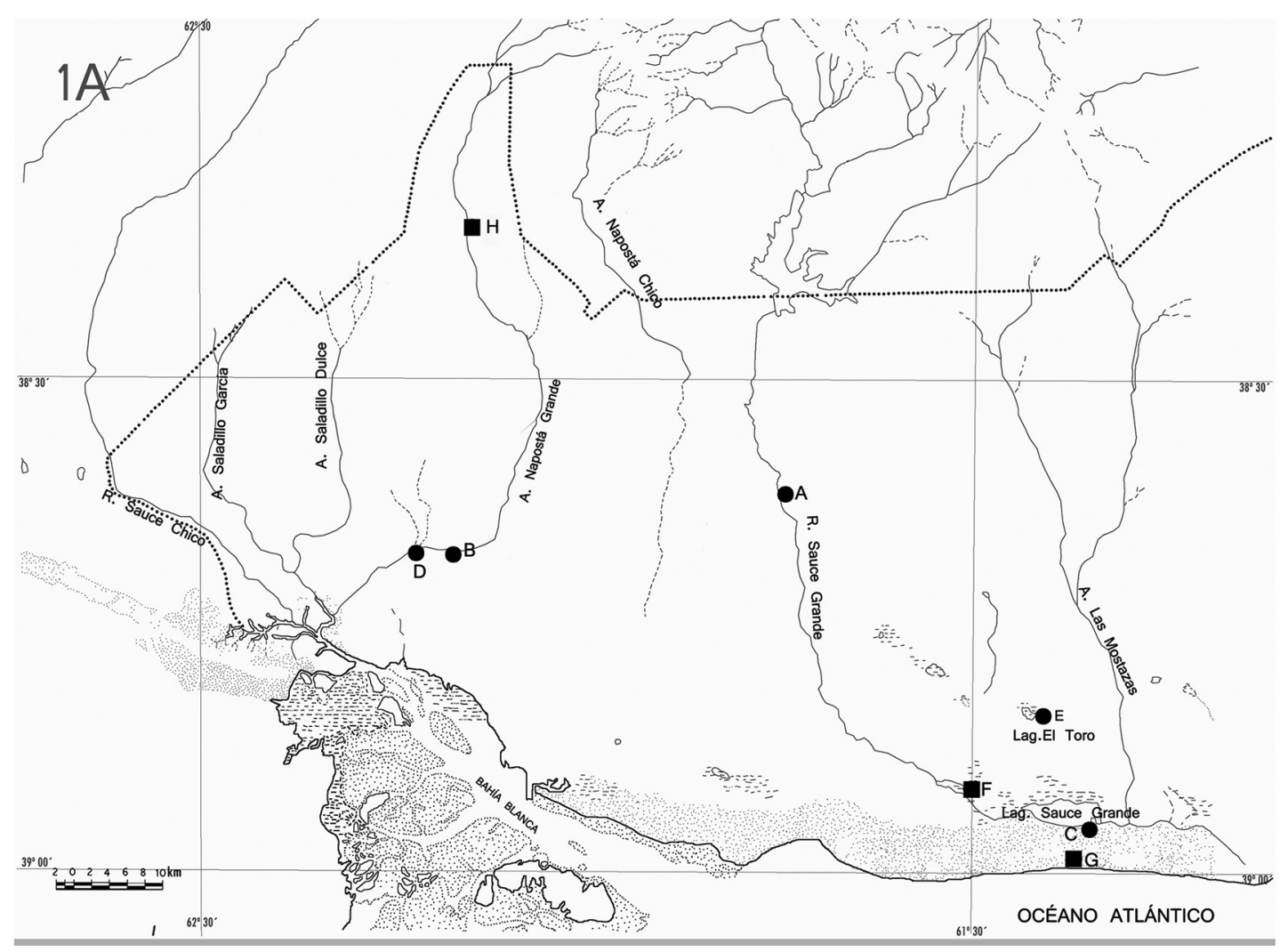

Figura 1A: Mapa con los sitios con isótopos estables humanos en toda la región. Referencias: (1-2) Laguna de Puán 1 y 6, (3-4) Laguna Los Chilenos 1 y 2, (5-6) Laguna Chasicó, (7) La Primavera, (8) Paso Alsina 1, (9) La Petrona, (10) Laguna Tres Reyes, (11) Campo Brochetto; (12) El Guanaco 1, (13) Túmulo de Malacara, (14) Laguna de Sotelo, (15) Laguna La Salada, (16) La Colorada, (17-19) La Guillerma Ñandú, La Guillerma 1 y 5, (20) Arroyo Fredes; (21-22) La Bellaca 1 y 2, (23) Arroyo Sarandí 2, (24) Anahi, (25) Arroyo Malo, (26) Garín, (27) Loma Chapalcó, (28-29) La Lomita, Tapera Moreira 3, (30) Lag. Chillué 1, (31) Chenque I, (32) Médano Petroquímica, (33) Puesto Hernández, (34) Laguna Chadilauquen, (35) Médano La Enriqueta.

Location map showing sites from the Pampean region with stable isotopes analysis on human remains. References: (1-2) Laguna de Puán 1 and 6, (3-4) Laguna Los Chilenos 1 and 2, (5-6) Laguna Chasicó; (7) La Primavera, (8) Paso Alsina 1, (9) La Petrona, (10) Laguna Tres Reyes, (11) Campo Brochetto; (12) El Guanaco 1, (13) Túmulo de Malacara, (14) Laguna de Sotelo, (15) Laguna La Salada, (16) La Colorada, (17-19) La Guillerma Nandú, La Guillerma 1 and 5, (20) Arroyo Fredes, (21-22) La Bellaca 1 and 2, (23) Arroyo Sarandí 2, (24) Anahi, (25) Arroyo Malo, (26) Garín, (27) Loma Chapalcó, (28-29) La Lomita, Tapera Moreira 3 , (30) Lag. Chillué 1, (31) Chenque I, (32) Médano Petroquímica, (33) Puesto Hernández, (34) Laguna Chadilauquen, (35) Médano La Enriqueta. 


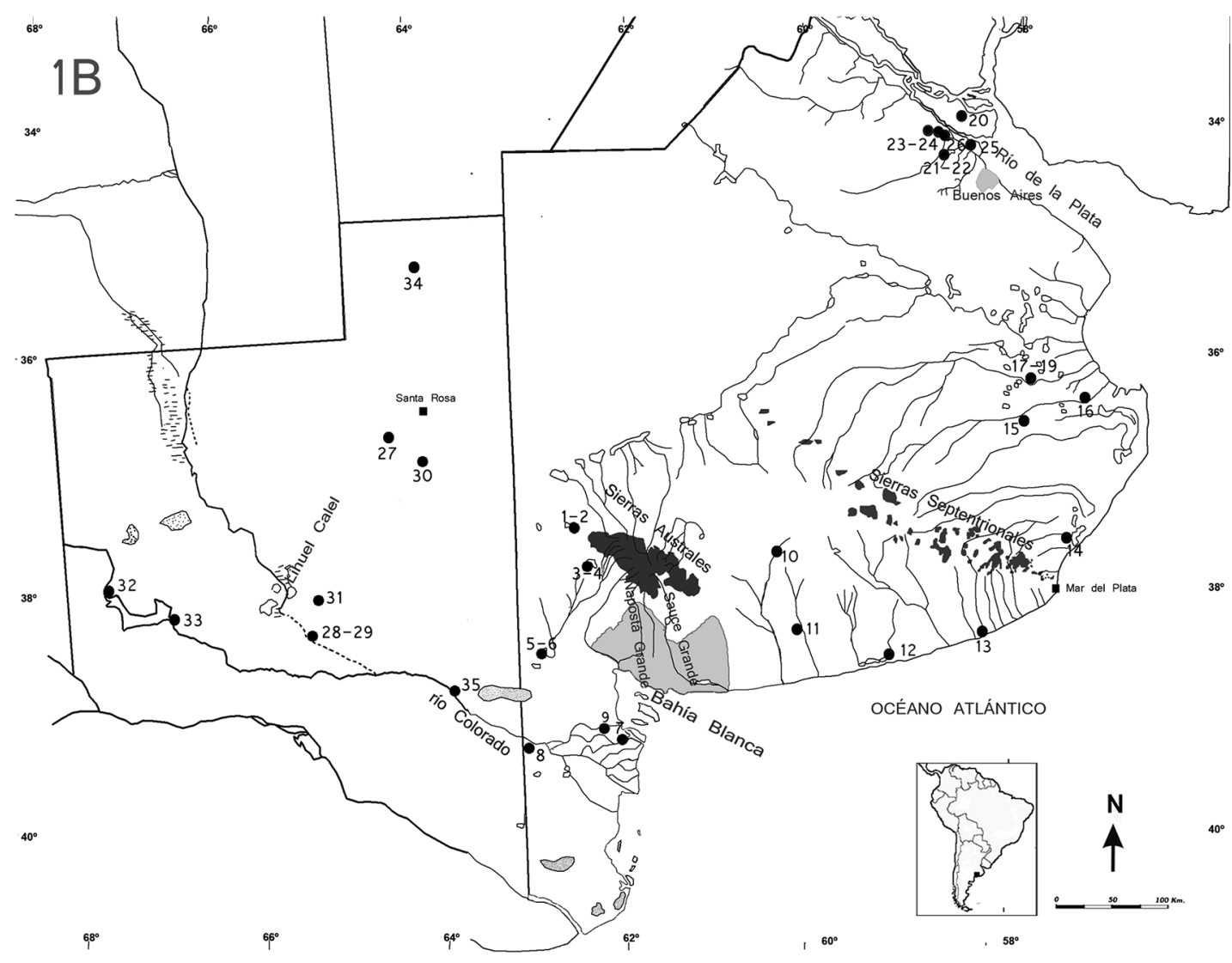

Figura 1B: Sector de trabajo delimitado por línea de puntos grises. Referencias: (a) sitio Paso Mayor Y1-2; (b) sitio Paso Vanoli; (c) sitio Laguna del Sauce; (d) sitio Villa Sapito; (e) sitio Laguna Toro; (f) sitio Puente de Fierro; (g) sitio Barrio Las Dunas; (h) sitio García del Río.

Location map with the analyzed sites outlined by gray dotted line. References: (a) site Paso Mayor Y1-2; (b) site Paso Vanoli; $(c)$ site Laguna del Sauce; $(d)$ site Villa Sapito; $(e)$ site Laguna Toro; $(f)$ site Puente de Fierro; $(g)$ site Barrio Las Dunas; $(h)$ site García del Río.

los vegetales, el origen marino o terrestre de los alimentos y la posición en la cadena trófica. Los análisis de $\delta^{13} \mathrm{C}$ se pueden realizar tanto sobre la fracción orgánica como sobre la inorgánica del hueso, obteniéndose en cada caso información diferente. Mientras que los valores isotópicos obtenidos del colágeno reflejan la dieta proteica, los efectuados sobre la apatita informan acerca de la dieta total (Ambrose y Norr 1993). Los procesos fotosintéticos se desarrollan de manera diferente según el tipo de planta y el ambiente. Las plantas con vía fotosintética C3 (Calvin Benson) se caracterizan por una mayor discriminación del ${ }^{13} \mathrm{C}$, dando como resultado valores de $\delta^{13} \mathrm{C}$ empobrecidos entre -35 y $-20 \%$ o. Por su parte, los vegetales con vía fotosintética C4 (Hatch-Slack) muestran valores más enriquecidos, entre -16 y $-7 \%$. Finalmente, las plantas CAM combinan vías fotosintéticas dando como resultado valores de $\delta^{13} \mathrm{C}$ que se superponen con los vegetales C3 y C4 (Ambrose 1993). De esta manera los herbívoros consumidores de vegetales $\mathrm{C} 3$ van a presentar valores medios de $-21 \%$ en el colágeno, en tanto que aquellos que basan su dieta en vegetales $\mathrm{C} 4$ van a mostrar valores más enriquecidos de aproximadamente $-7,5 \%$. En cuanto a los valores de apatita los herbívoros consumidores de vegetales $\mathrm{C} 4$ presentan valores medios de $-6 \%$ y los consumidores de vegetales C3 de -14\%o (Katzenberg 2008). 
Los animales marinos poseen valores de $\delta^{13} \mathrm{C}$ más enriquecidos que los animales terrestres y semejantes a los de los vegetales C4. Los análisis de $\delta^{15} \mathrm{~N}$ permiten conocer el nivel trófico de los individuos, ya que a medida que se asciende en la cadena trófica se da un fraccionamiento de 2 a $3 \%$. Además los alimentos marinos presentan valores de ${ }^{15} \mathrm{~N}$ más enriquecidos que los terrestres, ya que las cadenas tróficas son más largas y existe un mayor enriquecimiento en ${ }^{15} \mathrm{~N}$ en estos ambientes (Barberena 2014). De esta manera los individuos con dietas basadas en alimentos marinos van a presentar señales isotópicas de $\delta^{15} \mathrm{~N}$ más enriquecidas que aquellos con una alimentación terrestre.

Este trabajo se basa en la información isotópica sobre restos humanos y faunísticos que provienen de ocho sitios ubicados en diversos ambientes: Paso Mayor YI S1-2 (NS y NI), Paso Vanoli, Laguna del Sauce, Villa Sapito, Laguna Toro, García del Río, Puente de Fierro y Barrio Las Dunas (Figura 1B). Se analizarán 18 muestras, de las cuales cinco corresponden a restos humanos y 13 a materiales faunísticos, totalizando 26 datos isotópicos (Tablas 1 y 2).

Los restos humanos provienen de cinco contextos mortuorios diferentes, correspondientes al Holoceno Tardío y cubren un lapso entre 2.400 y 700 años a.p. (Tabla 1). En todos los casos los análisis se hicieron sobre fragmentos de costilla de individuos adultos. En estos restos se realizaron análisis isotópicos completos (de $\delta^{13} \mathrm{Ccol}$. y apat. y $\delta^{15} \mathrm{~N}$ ) y en un caso el valor de $\delta^{13} \mathrm{Ccol}$. es el informado junto con el fechado radiocarbónico. En tanto que para la fauna en cuatro muestras se obtuvieron valores de $\delta^{13} \mathrm{Ccol}$. específicos (Rhea americana, Anatidae, Myocastor coypus y Chaetophractus villosus); los restantes datos provienen de los valores de $\delta^{13} \mathrm{Ccol}$. informado junto con el fechado radiocarbónico. Los análisis isotópicos específicos se realizaron en los laboratorios especializados del Center for Applied Isotope Studies, University of Georgia (Estados Unidos).

\section{Resultados}

\section{Ecología isotópica del sector de estudio}

Para efectuar inferencias más confiables en relación con la dieta de los individuos, en primera instancia se analizaron los valores isotópicos de los recursos más abundantes en los sitios arqueológicos del sector (Tabla 2). Esta información provee el marco de referencia a partir del cual se pueden realizar las inferencias paleodietarias (Méndez et al. 2014; Zangrando et al. 2014). Para obtener un cuadro más completo y debido a la falta de datos propios de $\delta^{15} \mathrm{~N}$ para los restos faunísticos esta información es complementada con la publicada para la pampa bonaerense por otros investigadores (Tabla 3).

En el caso de los estudios isotópicos realizados por nosotros la información proviene de cinco sitios (Tabla 2). Las especies analizadas corresponden a herbívoros de gran porte-Lama guanicoe (guanaco) y Ozotoceros bezoarticus (venado)-; mamíferos de porte menor-Myocastor coypus (coipo) y Chaetophractus villosus (peludo); aves terrestres, Rhea americana (ñandú) y acuáticas (Anatidae, pato) y finalmente fauna marina -Otariidae y Pogonias cromis (corvina negra) (Tabla 2 ).

Los herbívoros de porte grande muestran un rango de valores de $\delta^{13} \mathrm{Ccol}$. entre $-20 \%$ o y $-18,6 \%$ o con un valor medio de $-19,5 \%$ o. Esto es lo esperable para herbívoros consumidores de vegetales $\mathrm{C} 3$. El rango de dispersión es de $1,4 \%$ o lo que indica que no hay diferencias significativas entre los valores de los herbívoros de los diferentes sitios y con distintas cronologías, por lo que se infiere que habrían consumido las mismas formaciones vegetales. En cuanto a otros recursos terrestres como los mamíferos de porte menor, el coipo presenta un valor de $\delta^{13} \mathrm{C}$ ligeramente más empobrecido que los herbívoros de porte grande $(-21,6 \%)$, reflejando también el consumo de vegetales de tipo C3. Por otro lado el peludo presenta valores de $\delta^{13} \mathrm{C}(-15,5 \%$ ) que indican el consumo de algún alimento enriquecido en ${ }^{13} \mathrm{C}$ y podría estar reflejando el carácter omnívoro de la dieta de estos animales.

En lo que respecta a las aves, el ñandú presenta un $\delta^{13} \mathrm{C}$ de $-20 \%$ o que revela el consumo de vegetales C3 y se superpone con los valores de otros herbívoros terrestres, mientras que los anátidos muestran $\delta^{13} \mathrm{C}$ más enriquecidos; estos valores de $-15 \%$ o son coherentes para aves de ambientes lagunares y se solapan con el obtenido para el peludo. Finalmente, con los valores más enriquecidos en ${ }^{13} \mathrm{C}$ (entre $-12,7$ y $-12,3 \%$ o) tenemos los recursos marinos que incluyen al lobo marino y a la corvina negra.

Tomando en cuenta todos los valores de $\delta^{13} \mathrm{Ccol}$. de la fauna del sector de estudio vemos que isotópicamente se distinguen: en un extremo los 
Tabla 1. Valores isotópicos e información bioarqueológica de los restos humanos. Isotopic values and bioarchaeological information of human remains.

\begin{tabular}{|c|c|c|c|c|c|c|c|}
\hline Sitio/distancia al mar & Años a.p. & $\delta^{13} \mathrm{C} \mathrm{col}$ & $\delta^{13} \mathrm{C}$ ap. & $\Delta \delta^{13} \mathrm{C}$ & $\delta^{15} \mathrm{~N}$ & Código laboratorio & Referencias \\
\hline $\begin{array}{c}\text { Paso Mayor YI S2 } \\
41 \mathrm{~km} \text { al mar }\end{array}$ & $700 \pm 40$ & $-18,7$ & $-14,1$ & 4,6 & 11,8 & UGAMS-16596 & $\begin{array}{c}\text { Bayón et al. 2010; } \\
\text { En este trabajo }\end{array}$ \\
\hline $\begin{array}{l}\text { Paso Vanoli } \\
13 \mathrm{~km} \text { al mar }\end{array}$ & $922 \pm 46$ & $-17,8$ & - & - & - & AA-106111 & En este trabajo \\
\hline $\begin{array}{l}\text { Laguna del Sauce } \\
5 \mathrm{~km} \text { al mar }\end{array}$ & $1.959 \pm 42$ & $-16,9$ & $-15,5$ & 1,4 & 11,5 & $\begin{array}{c}\text { AA-56778 } \\
\text { UGAMS-16602 }\end{array}$ & En este trabajo \\
\hline $\begin{array}{l}\text { Villa Sapito } \\
7 \mathrm{~km} \text { al mar }\end{array}$ & $2.306 \pm 41$ & $-17,3$ & $-11,2$ & 6,1 & 12,7 & UGAMS-16595 & En este trabajo \\
\hline $\begin{array}{l}\text { Laguna Toro } \\
12 \mathrm{~km} \text { al mar }\end{array}$ & $2.369 \pm 52$ & $-16,8$ & $-13,5$ & 3,3 & 11,8 & UGAMS-16601 & En este trabajo \\
\hline Máximo/Mínimo & - & $\begin{array}{c}-16,8 \text { a } \\
-18,7\end{array}$ & $\begin{array}{c}-11,2 \mathrm{a} \\
-15,5\end{array}$ & - & $\begin{array}{c}12,7 \mathrm{a} \\
11,5\end{array}$ & - & \\
\hline Media & - & $-17,4$ & $-13,5$ & 4,3 & 12 & - & \\
\hline
\end{tabular}

Tabla 2. Valores isotópicos de $\delta^{13} \mathrm{C}$ (col.) de los recursos faunísticos del área. $\delta^{13} \mathrm{C}$ (col.) values of fauna from the study area.

\begin{tabular}{|c|c|c|c|c|c|}
\hline Sitio y coordenadas & $\mathrm{msm}$ & Especie y elemento & $\delta^{13} \mathrm{C} \mathrm{col}$ & $\begin{array}{c}\text { Años a.p./ Código } \\
\text { Muestra }\end{array}$ & Referencias \\
\hline $\begin{array}{c}\text { García del Río } \\
\left(38^{\circ} 21^{\prime} \mathrm{S}, 62^{\circ} 9^{\prime} \mathrm{O}\right)\end{array}$ & 61 & $\begin{array}{l}\text { Lama guanicoe } \\
\text { (unciforme) }\end{array}$ & $-18,6$ & $\begin{array}{l}2.342 \pm 47 \\
\text { AA-71655 }\end{array}$ & Tomassini et al. 2014 \\
\hline $\begin{array}{c}\text { Paso Vanoli } \\
\left(38^{\circ} 40^{\prime} \mathrm{S}, 62^{\circ} 13^{\prime} \mathrm{O}\right)\end{array}$ & 45 & $\begin{array}{l}\text { Lama guanicoe } \\
\text { (unciforme) }\end{array}$ & $-19,5$ & $\begin{array}{c}714 \pm 53 \\
\text { AA-91414 }\end{array}$ & Vecchi et al. 2013 \\
\hline $\begin{array}{c}\text { Paso Mayor YI S1-2 } \\
\left(38^{\circ} 36^{\prime} \mathrm{S}, 61^{\circ} 44^{\prime} \mathrm{O}\right)\end{array}$ & 100 & $\begin{array}{c}\text { Lama guanicoe } \\
\text { (diáfisis hueso largo) }\end{array}$ & $-19,5$ & $\begin{array}{l}5.877 \pm 63 \\
\text { AA- } 71656\end{array}$ & Bayón et al. 2010 \\
\hline $\begin{array}{c}\text { Paso Mayor YI S1-2 } \\
\left(38^{\circ} 36^{\prime} \mathrm{S}, 61^{\circ} 44^{\prime} \mathrm{O}\right)\end{array}$ & 100 & $\begin{array}{l}\text { Lama guanicoe } \\
\text { (calcáneo) }\end{array}$ & $-19,8$ & $\begin{array}{l}3.820 \pm 47 \\
\text { AA- } 82709\end{array}$ & Bayón et al. 2010 \\
\hline $\begin{array}{l}\text { Paso Mayor YI S1-2 } \\
\left(38^{\circ} 36^{\prime} \mathrm{S}, 61^{\circ} 44^{\prime} \mathrm{O}\right)\end{array}$ & 100 & $\begin{array}{l}\text { Lama guanicoe } \\
\text { (falange) }\end{array}$ & $-19,9$ & $\begin{array}{l}4.046 \pm 57 \\
\text { AA- } 82709\end{array}$ & Bayón et al. 2010 \\
\hline $\begin{array}{l}\text { Paso Mayor YI S1-2 } \\
\left(38^{\circ} 36^{\prime} \mathrm{S}, 61^{\circ} 44^{\prime} \mathrm{O}\right)\end{array}$ & 100 & $\begin{array}{l}\text { Lama guanicoe } \\
\text { (metápodo) }\end{array}$ & -19 & $\begin{array}{l}2.774 \pm 45 \\
\text { AA- } 82714\end{array}$ & Bayón et al. 2010 \\
\hline $\begin{array}{c}\text { Paso Mayor YI S1-2 } \\
\left(38^{\circ} 36^{\prime} \mathrm{S}, 61^{\circ} 44^{\prime} \mathrm{O}\right)\end{array}$ & 100 & $\begin{array}{l}\text { Rhea americana } \\
\text { (tibiotarso) }\end{array}$ & $-20,1$ & UGAMS-16597 & Este trabajo \\
\hline $\begin{array}{c}\text { Puente de Fierro } \\
\left(38^{\circ} 56^{\prime} \mathrm{S}-61^{\circ} 27^{\prime} \mathrm{O}\right)\end{array}$ & 11 & $\begin{array}{l}\text { Anatidae } \\
\text { (cúbito) }\end{array}$ & -15 & UGAMS-16600 & Este trabajo \\
\hline $\begin{array}{c}\text { Puente de Fierro } \\
\left(38^{\circ} 56^{\prime} \mathrm{S}-61^{\circ} 27^{\prime} \mathrm{O}\right)\end{array}$ & 11 & $\begin{array}{c}\text { Ozotoceros bezoarticus } \\
\text { (falange) }\end{array}$ & -20 & $\begin{array}{l}2.042 \pm 49 \\
\text { AA- } 82715\end{array}$ & Frontini y Bayón 2015b \\
\hline $\begin{array}{c}\text { Puente de Fierro } \\
\left(38^{\circ} 56^{\prime} \mathrm{S}-61^{\circ} 27^{\prime} \mathrm{O}\right)\end{array}$ & 11 & $\begin{array}{l}\text { Myocastor coypus } \\
\text { (fémur) }\end{array}$ & $-21,6$ & UGAMS-16598 & Este trabajo \\
\hline $\begin{array}{c}\text { Puente de Fierro } \\
\left(38^{\circ} 56^{\prime} \mathrm{S}-61^{\circ} 27^{\prime} \mathrm{O}\right)\end{array}$ & 11 & $\begin{array}{c}\text { Chaetophractus villosus } \\
\text { (húmero) }\end{array}$ & $-15,5$ & UGAMS-16599 & Este trabajo \\
\hline $\begin{array}{l}\text { Barrio Las Dunas } \\
\left(38^{\circ} 59^{\prime} \mathrm{S}, 61^{\circ} 20^{\prime} \mathrm{O}\right)\end{array}$ & 9 & $\begin{array}{l}\text { Otariidae } \\
\text { (húmero) }\end{array}$ & $-12,3$ & $\begin{array}{l}6.924 \pm 69 \\
\text { AA-71654 }\end{array}$ & Bayón et al. 2012 \\
\hline $\begin{array}{l}\text { Barrio Las Dunas } \\
\left(38^{\circ} 59^{\prime} \mathrm{S}, 61^{\circ} 20^{\prime} \mathrm{O}\right)\end{array}$ & 9 & $\begin{array}{l}\text { Pogonias cromis } \\
\text { (radio espinoso) }\end{array}$ & $-12,7$ & $\begin{array}{l}6.820 \pm 100 \\
\text { AA-93961 }\end{array}$ & Bayón et al. 2012 \\
\hline
\end{tabular}

animales marinos con los valores más enriquecidos en ${ }^{13} \mathrm{C}$, en el otro extremo y con los valores más empobrecidos se encuentra el coipo, el ñandú y los herbívoros terrestres de porte grande, cuyos valores isotópicos son indistinguibles. Finalmente, con valores intermedios están las aves acuáticas y el peludo.

Cuando comparamos nuestros datos isotópicos con los disponibles para otros sitios de la región (Tabla 3) se observa que en el caso de los mamíferos 
Tabla 3. Datos isotópicos de los recursos faunísticos de la región pampeana. Isotopic data of faunistic resources of the Pampean region.

\begin{tabular}{|c|c|c|c|c|}
\hline Ubicación/ Sitio & Especie & $\delta^{13} \mathrm{C} \mathrm{col}$ & $\delta^{15} \mathrm{~N}$ & Referencias \\
\hline El Tigre & Lama guanicoe & $\begin{array}{l}-19,9 \\
-20,1 \\
-20,2\end{array}$ & & Martínez et al. 2009 \\
\hline Loma Ruiz & Lama guanicoe & $\begin{array}{l}-17,8 \\
-16,2\end{array}$ & & Martínez et al. 2009 \\
\hline San Antonio 1 & Lama guanicoe & $-19,9$ & & Martínez et al. 2009 \\
\hline San Antonio 2 & Lama guanicoe & $\begin{array}{l}-20,9 \\
-19,7\end{array}$ & & Martínez et al. 2009 \\
\hline Valle inferior río Colorado & $\begin{array}{l}\text { Ozotoceros bezoarticus } \\
\text { Rhea americana }\end{array}$ & $\begin{array}{c}-22,12 \\
-21,1 \\
-21,06 \\
-22,25\end{array}$ & 4,36 & $\begin{array}{l}\text { Martínez et al. } 2009 \\
\text { Martínez et al. } 2009\end{array}$ \\
\hline La Olla & Otariidae & $\begin{array}{c}-13,7 \\
-12,3 \\
-12 \\
-11,5\end{array}$ & $\begin{array}{l}21,1 \\
21,1\end{array}$ & Politis et al. 2009 \\
\hline Quequén Salado 1 & Lama guanicoe & $\begin{array}{c}-18,6 \\
-19 \\
-19,1 \\
-18,9\end{array}$ & & Barberena et al. 2009 \\
\hline Quequén Salado 2 & Lama guanicoe & $-19,3$ & & Barberena et al. 2009 \\
\hline Arroyo Seco 2 & Lama guanicoe & $\begin{array}{l}-19,8 \\
-23,3\end{array}$ & & Politis et al. 2009 \\
\hline Tres Reyes 1 & $\begin{array}{l}\text { Ozotoceros bezoarticus } \\
\text { Lama guanicoe }\end{array}$ & $\begin{array}{l}-19,4 \\
-20,4 \\
-19,9\end{array}$ & 5,9 & $\begin{array}{c}\text { Politis et al. } 2009 \\
\text { Madrid y Barrientos } 2000\end{array}$ \\
\hline Zanjón Seco 2 & Lama guanicoe & $\begin{array}{l}-19,5 \\
-19,8\end{array}$ & $\begin{array}{l}6,3 \\
5,6\end{array}$ & Barberena et al. 2009 \\
\hline $\begin{array}{c}\text { Paso Otero } 1 \\
\text { Cortaderas }\end{array}$ & $\begin{array}{l}\text { Lama guanicoe } \\
\text { Lama guanicoe }\end{array}$ & $\begin{array}{c}-19 \\
-23.6\end{array}$ & & $\begin{array}{l}\text { Barberena et al. } 2009 \\
\text { Barberena et al. } 2009\end{array}$ \\
\hline Nutria Mansa 1 & Lama guanicoe & $\begin{array}{l}-25 \\
-25 \\
-25,3 \\
-24,6\end{array}$ & & Bonomo 2005 \\
\hline Calera & Lama guanicoe & $\begin{array}{l}-18,5 \\
-18,8 \\
-19,2 \\
-20,8 \\
-22,6 \\
-19,1\end{array}$ & & Barberena et al. 2009 \\
\hline La Barrancosa & Lama guanicoe & $-20,2$ & & Barberena et al. 2009 \\
\hline Alfar & Otariidae & $-11,5$ & & Barberena et al. 2009 \\
\hline La Guillerma 1 & Myocastor coypus & $-15,9$ & 4,5 & Scabuzzo y González 2007 \\
\hline Guazunambí & Ozotoceros bezoarticus & $-18,8$ & 5,23 & Loponte y Acosta 2007 \\
\hline Garín & Myocastor coypus & $-17,9$ & 4,3 & Loponte y Acosta 2007 \\
\hline
\end{tabular}

terrestres de tamaño grande (guanaco y venado) los valores de $\delta^{13} \mathrm{Ccol}$. aquí presentados entran dentro del rango de valores disponibles para los otros sitios (media $=-20,4 \%$ ). Se cuenta con cinco valores de $\delta^{15} \mathrm{~N}$ que muestran un valor medio de $5,65 \%$ 。 (sitios Arroyo Seco 2, Zanjón Seco 2, Guazunambí y valle inferior del río Colorado) (Tabla 3). En cuanto a la fauna de porte menor hay dos datos de $\delta^{13} \mathrm{Ccol}$. de coipo procedentes de los sitios $\mathrm{La}$ Guillerma 1 y Garín que son más enriquecidos que los presentados aquí. Para estas mismas muestras se obtuvieron dos valores de $\delta^{15} \mathrm{~N}$ de $4,4 \%$ en 
promedio. Para las aves terrestres en el valle inferior del río Colorado se obtuvieron tres valores de $\delta^{13} \mathrm{Ccol}$. (media $=-21,4 \%$ ) muy semejantes a los presentados aquí y que en ambos casos reflejan el consumo de vegetales C3 (Martínez et al. 2009). Para una de estas muestras se cuenta con un valor de $\delta^{15} \mathrm{~N}$ de $4,3 \%$ o. Finalmente, a nivel regional se conocen cinco datos de $\delta^{13} \mathrm{Ccol}$. de lobo marino procedentes de dos sitios (La Olla y Alfar) con un valor medio de $-12,3 \%$ o que es indistinguible del valor presentado para el sudoeste. Adicionalmente en dos muestras se realizaron estudios de $\delta^{15} \mathrm{~N}$ que arrojaron un valor de $21,1 \%$.

\section{Los restos humanos}

Los valores de $\delta^{13} \mathrm{Ccol}$. obtenidos varían entre $-18,7$ y $-16,8 \%$ o (media $=-17,4 \%$ o (Tabla 1). Esto indica que la dieta de los individuos estuvo basada en la ingesta de animales terrestres consumidores de vegetales $\mathrm{C} 3$, que incluyen al guanaco, venado, coipo y ñandú, siendo en especial el individuo del sitio Paso Mayor el que presenta los valores de colágeno más empobrecidos. Sin embargo, los valores más enriquecidos de los individuos de los sitios Villa Sapito, Paso Vanoli, Laguna el Toro y Laguna del Sauce entrarían dentro del rango de dietas mixtas, que además de los herbívoros consumidores de C3 habrían incorporado alimentos más enriquecidos como podrían ser los de origen marino, el peludo o las aves acuáticas (patos) (Figura 2). Por su parte, los valores de $\delta^{15} \mathrm{~N}$ comprenden un rango entre $11,5 \%$ y $12,7 \%$ o (media $=12 \%$ o, Tabla 1$)$. Estos valores elevados reflejan un importante componente cárnico en la dieta. Esto además se ve apoyado por la diferencia entre la fracción orgánica e inorgánica (expresado como $\Delta \delta^{13} \mathrm{C}$ ) con un promedio de $4,3 \%$. En la Figura 3 se presenta la dispersión de los valores de $\delta^{13} \mathrm{Ccol}$. y $\delta^{15} \mathrm{~N}$ de las muestras humanas y de distintos recursos faunísticos de la región. Como se observa en la figura el $\delta^{15} \mathrm{~N}$ de los humanos se encuentran enriquecidos más de seis partes por mil respecto de los valores medios de $\delta^{15} \mathrm{~N}$ de herbívoros terrestres $\left(\delta^{15} \mathrm{~N}=5,07 \%\right.$ o, Tabla 3, Figura 2$)$. Esta diferencia en los valores de $\delta^{15} \mathrm{~N}$ de los humanos y los herbívoros terrestres no se puede explicar solo por un incremento de los valores por el aumento en los niveles tróficos y podría estar indicando el consumo de otros recursos enriquecidos en ${ }^{15} \mathrm{~N}$ (Martínez et al. 2009). En cuanto a los valores de apatita, los mismos comprenden un rango entre
$-15,5$ y $-11,2 \%$ o con una media de $-13,5 \%$ o. Estos son los valores esperados para dietas terrestres basadas en el consumo de vegetales $\mathrm{C} 3\left(\delta^{13} \mathrm{C}=-19\right.$ $\mathrm{a}-14 \%$ o) y mixtas que combinan distintas cantidades de alimentos marinos y terrestres $\left(\delta^{13} \mathrm{C}\right.$ (apatita $)=$ -15 a $-11 \%$, Barberena 2002).

\section{Discusión}

Si bien la muestra humana analizada es pequeña, permite realizar algunas inferencias de interés y abrir una nueva agenda de trabajo. El estudio isotópico reflejó que todos los individuos tuvieron dietas cárnicas, esto se vio tanto en los valores elevados de nitrógeno como en la diferencia entre el colágeno y la apatita del carbono. Predominantemente, las proteínas se obtuvieron de distintos animales terrestres, aunque no se descarta el consumo en pequeña escala de alimentos marinos; esto es coherente con algunos valores de $\delta^{13} \mathrm{Ccol}$. que indican dietas mixtas y con los valores de $\delta^{15} \mathrm{~N}$. Sin embargo hay que considerar también que otros factores pudieron influir en el aumento del $\delta^{15} \mathrm{~N}$, como el estrés hídrico o el consumo de algún otro recurso terrestre enriquecido en ${ }^{15} \mathrm{~N}$, no detectado hasta ahora. Para evaluar estas alternativas resulta necesario realizar análisis de $\delta^{15} \mathrm{~N}$ sobre los distintos recursos consumidos en el sector de estudio (Zangrando et al. 2014).

Una línea de evidencia independiente, como es el registro arqueofaunístico, permite cotejar las decisiones respecto de la alimentación. Los datos zooarqueológicos de los sitios bajo estudio dan cuenta de un predominio del guanaco, como presa principal, complementada con otros herbívoros terrestres (venado de las pampas, ñandú, coipo) y en menor proporción con peludo, aves acuáticas y dos especies de otáridos. Específicamente, la presencia de restos de Arctocephalus australis y Otaria flavescens en uno de los sitios del interior (PF), muestra el traslado de estas presas por $7 \mathrm{~km}$ y el consumo en este campamento residencial.

A su vez, la representación de alimentos marinos en las arqueofaunas se puede relacionar, en términos de movilidad, con el traslado de otros recursos litorales como los rodados costeros y los moluscos que fueron transportados hacia todos los sitios tardíos del sector. De este modo, distintos registros indican la circulación reiterada de los grupos entre el interior y la costa (Frontini y Bayón 2015a). 




Figura 2. Gráfico valores de $\delta^{13} \mathrm{C}$ (col) de los humanos y de la fauna. $\delta^{13} C$ (collagen) values of human and faunal remains.

Desde una perspectiva espacial, los temas de la distancia al mar y la ocurrencia de recursos marinos (líticos y faunísticos) en sitios del interior de la región pampeana han sido analizados por otros autores en relación con los sistemas de movilidad y los territorios (Bonomo 2005; Politis et al. 2003). En este sentido, se propuso un decrecimiento en la importancia de los recursos litorales, sobre todo líticos, a medida que se incrementa la distancia a la costa (Bonomo 2005; Politis et al. 2003). Los datos presentados previamente para el área de trabajo basados en el traslado desde la costa de recursos faunísticos y líticos serían, en términos generales, coincidentes con esta propuesta (Frontini y Bayón 2015a).

A continuación compararemos nuestros datos isotópicos con aquellos generados para otros sitios de la región pampeana correspondientes al Holoceno Tardío. El estado actual del conocimiento incluye estudios paleodietarios de 34 sitios, sobre la base de análisis isotópicos en restos humanos, en la Figura 4 se muestra para cada área una correlación de los valores de $\delta^{13} \mathrm{C}$ y $\delta^{15} \mathrm{~N}$, efectuada sobre los restos humanos que presentan ambos resultados. En el área Sur, adyacente a nuestro sector de trabajo, a partir de los valores isotópicos de $\delta^{13} \mathrm{Ccol}$. (entre $-20,9$ y $-16,2 \%$ ) y $\delta^{15} \mathrm{~N}$ (entre 10,3 y $14,1 \%$ ) se infirió la ingesta de alimentos continentales y en algunos sitios la incorporación de peces marinos y fluviales (Barrientos et al. 2015; Martínez et al. 2009). Los datos presentados por Martínez et al. (2009) muestran que en el valle inferior del río Colorado, los rangos isotópicos son más amplios que los aquí presentados tanto para el $\delta^{13} \mathrm{Ccol}$. como para el $\delta^{15} \mathrm{~N}$. Por su parte, recientemente Barrientos et al. (2015) publicaron los resultados isotópicos de seis sitios ubicados en sectores colindantes (hacia el norte y el oeste) al de este trabajo. En este caso los valores de $\delta^{13} \mathrm{Ccol}$. son muy semejantes a los del sector de trabajo, aunque se observa una diferencia 

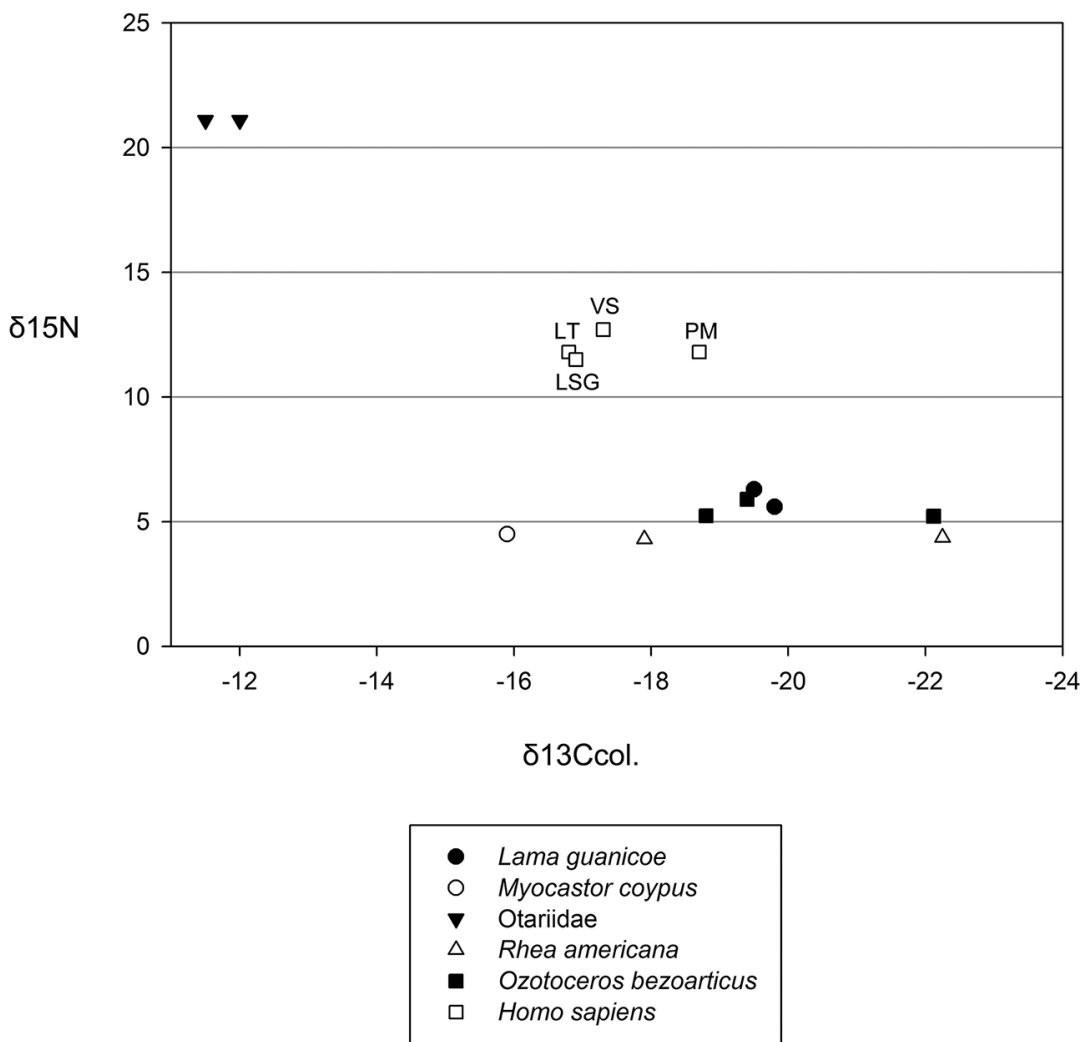

Figura 3. Dispersión de los valores de $\delta^{13} \mathrm{Ccol}$. y $\delta^{15} \mathrm{~N}$ de las muestras humanas del sector y de distintos recursos faunísticos de la región pampeana. Referencias: (LT) Laguna Toro, (VS) Villa Sapito, (LSG) Laguna del Sauce, (PM) Paso Mayor.

$\delta^{13} \mathrm{Ccol}$. and $\delta^{15} \mathrm{~N}$ values of human samples from study area and regional faunal resources. References: $(L T)$ Laguna Toro, (VS) Villa Sapito, (LSG) Laguna del Sauce, (PM) Paso Mayor.

en algunos valores de $\delta^{15} \mathrm{~N}$ que tienden a ser más bajos (media $=11,1 \%$ o).

Hacia el este, en el área Interserrana bonaerense se publicaron los resultados isotópicos de cuatro sitios (Barrientos et al. 2015; Flegenheimer et al. 2002; Madrid y Barrientos 2000; Politis et al. 2011). Los valores de colágeno (entre $-18,5$ y $-17,2$ media $=-17,9 \%$ ) se superponen con los presentados aquí, mientras que los de $\delta^{15} \mathrm{~N}(11,9$ y $12 \%$ ) son semejantes al promedio de nuestras muestras. Las dietas fueron interpretadas como continentales y mixtas, con una pequeña proporción de alimentos marinos. En la Depresión del río Salado la información isotópica proviene de seis sitios, los datos de $\delta^{13} \mathrm{Ccol}$. (rango entre $-19,5 \mathrm{y}-14,2 \%$ ) y $\delta^{15} \mathrm{~N}$ (entre 9,2 y $12,3 \%$ ) son más amplios que los sitios aquí presentados e indican que coexistieron dietas mixtas y continentales, con el consumo de recursos lagunares (Murgo y Aldazabal 2007; Scabuzzo y González 2007). En el área norte la alimentación se basó en la ingesta de recursos continentales y en algunos casos se incorporaron vegetales del tipo C4 (Loponte y Acosta 2007), los datos de $\delta^{13} \mathrm{Ccol}$. presentados para el área tienen un rango entre $-20,3 \mathrm{y}-15,1 \%$ y para $\delta^{15} \mathrm{~N}$ entre 8,1 y $11,2 \%$ o. Finalmente, para la Pampa Seca los datos $\left(\delta^{13} \mathrm{Ccol}\right.$. entre $-21,2$ y $-15,7 \%$ y $\delta^{15} \mathrm{~N}$ entre 10 y $16,3 \%$ ) señalan dietas continentales y mixtas (Berón et al. 2009) y en algunos casos los valores de apatita podrían reflejar el consumo de vegetales C4 o CAM (Mendonça et al. 2013).

En síntesis, los valores obtenidos en este trabajo de $\delta^{13} \mathrm{Ccol}$. entre $-18,7$ a $-16,8 \%$ y de $\delta^{15} \mathrm{~N}$ entre 11,5 y $12,7 \%$ o se encuentran dentro del rango de valores publicados para los sectores contiguos tanto hacia el sur como hacia el este. Finalmente, a nivel 


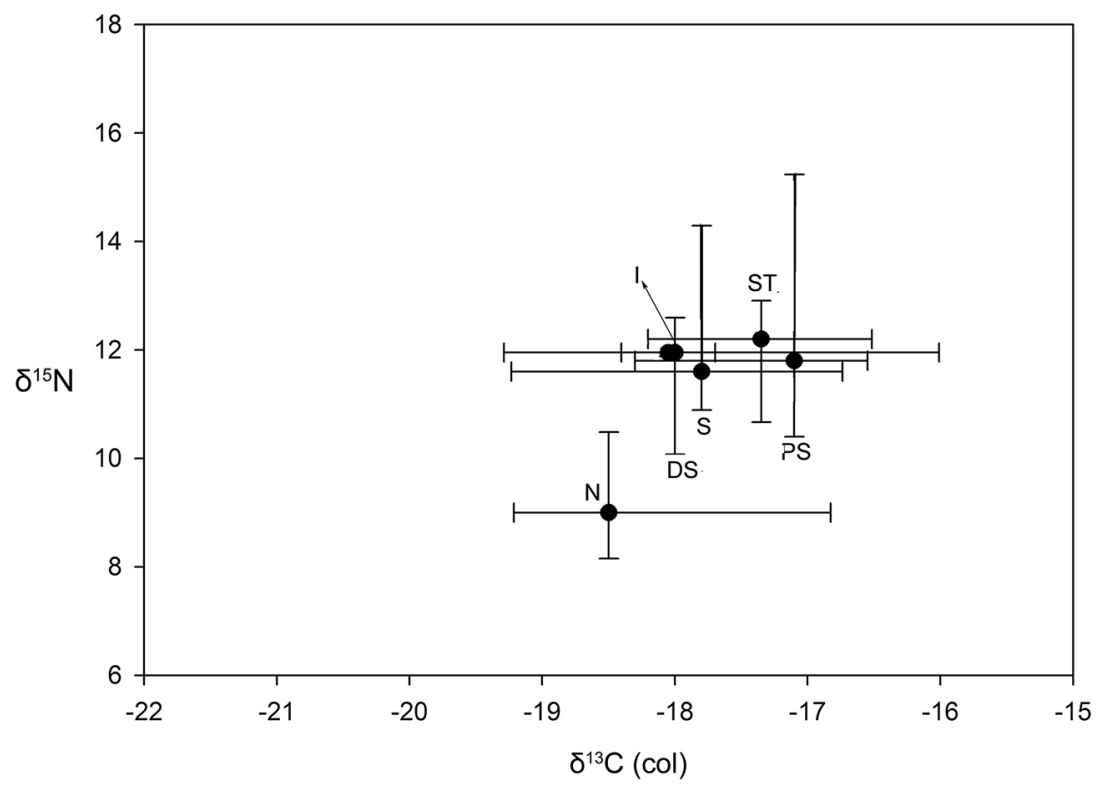

Figura 4. Correlación de los valores de $\delta^{13} \mathrm{C}$ y $\delta^{15} \mathrm{~N}$ de los restos humanos de cada área de la Región Pampeana. Referencias: (SW) Sector de estudio, (I) área Interserrana, (DS) Depresión del río Salado, $(\mathrm{S})$ área Sur, (PS) Pampa Seca, $(\mathrm{N})$ área Norte.

Correlation of ( $\delta^{13} \mathrm{C}$ and $\left(\delta^{15} \mathrm{~N}\right.$ values of human remains from the Pampean region. References: $(S W)$ study area, (I) Interserrana area, (DS) the río Salado Depression, (S) southern area, (PS) Pampa Seca, $(N)$ northern area.

regional se destaca una gran variabilidad en los tipos de recursos consumidos entre las áreas geográficas y en el interior de cada una de ellas. Los valores más distantes de los aquí presentados corresponden al área norte (Figura 4).

\section{Conclusiones}

Este trabajo representa el primer aporte al estudio paleodietario mediante isótopos estables del sector bajo estudio en el sudoeste bonaerense. También se informan cuatro nuevas dataciones radiocarbónicas sobre restos humanos de cuatro sitios diferentes, conjuntamente con los 13 nuevos valores isotópicos realizados sobre los esqueletos.

Los estudios paleodietarios por medio de isótopos estables en el área de investigación sirvieron como una valiosa línea de evidencia para conocer la subsistencia, la movilidad y aportar al entendimiento de la explotación de los diferentes ambientes. El análisis de la dieta de los individuos indica el consumo de proteínas provenientes de herbívoros de porte grande y pequeño sumado a aves acuáticas y terrestres, a lo que probablemente se agregó la ingesta de alimento marino en pequeñas cantidades. El registro arqueofaunístico muestra el consumo de guanaco, venado de las pampas, ñandú, peludo, coipo, pato y lobo marino. Esta selección de presas es concordante con la información isotópica en restos humanos. En cuanto a la movilidad, la circulación entre el interior y la costa como una práctica rutinaria de los grupos cazadores recolectores está avalada por diversos registros: faunísticos, tecnológicos e isotópicos.

En relación con la reconstrucción de una ecología isotópica para el área, la investigación se inició incluyendo aquellos animales que, desde el registro arqueofaunístico, evidenciaban consumo. No obstante, como el área de estudio es un ecotono donde confluyen recursos de distintos ambientes con una gran diversidad de presas se considera necesario analizar una mayor variedad de especies, de obtener valores de una misma especie en distintos entornos y de incluir resultados de $\delta^{15} \mathrm{~N}$ que no se han realizado hasta el momento. La obtención de nuevos datos ayudará a una mejor comprensión de las paleodietas de las poblaciones que habitaron el sector durante el Holoceno Tardío. 
Agradecimientos: Los trabajos fueron financiados por los subsidios SECYT-UNS 24/I 182, Préstamo BID PICT-20130179 y Préstamo BID PICT-20132175. Agradecemos a: Reserva Natural Provincial Pehuenco-Monte Hermoso; Museo Municipal de Monte Hermoso y Museo y Archivo Histórico
Municipal de Bahía Blanca; a la Sra. Silvia Bilbao de González Martínez e hijos por permitirnos el acceso a Puente de Fierro. Finalmente, a los dos revisores anónimos que ayudaron a mejorar sustancialmente el texto. El contenido del trabajo es absoluta responsabilidad de los autores.

\section{Referencias Citadas}

Ambrose, A. 1993. Isotopic analysis of paleodiets: Methodological and interpretative considerations. En Investigations of Ancient Human Tissue. Chemical Analyses in Anthropology, editado por M. Standford, pp. 59-130. Gordon and Breach Science Publishers, Pennsylvania.

Ambrose, S. y L. Norr 1993. Experimental evidence for the relationship of the carbon isotope ratios of whole diet and dietary protein to those of bone collagen and carbonate. En Prehistoric Human Bone: Archaeology at the Molecular Level, editado por J.B. Lambert y G. Grupe, pp. 1-37. SpringerVerlag, New York.

Austral, A. 1968. Prehistoria de la Región Pampeana Sur. Universidad Nacional del Sur, Bahía Blanca.

Bayón, C., R. Frontini y R. Vecchi 2012. Middle holocene settlements in coastal dunes from southwest of Buenos Aires province, Argentina. Quaternary International 256:54-61.

Bayón, C., A. Pupio, R. Frontini, R. Vecchi y C. Scabuzzo 2010. Localidad Arqueológica Paso Mayor: nuevos estudios 40 años después. Intersecciones en Antropología 11:155-166.

Barberena, R. 2002. Los Límites del Mar. Isótopos Estables en Patagonia Meridional. Sociedad Argentina de Antropología, Buenos Aires.

Barberena, R. 2014. Isotopicstudies of foragers' diet: Environmentalarchaeologicalapproaches. En Encyclopedia of Global Archaeology, editada por C. Smith, pp. 4111-4120. Springer.

Barberena, R., A. Zangrando, A. Gil, G. Martínez, G. Politis, L. Borrero y G. Neme 2009. Guanaco (Lama guanicoe) isotopic ecology in southern South America: spatial and temporal tendencies, and archaeological implications. Journal of Archaeological Science 36:2666-2675.

Barrientos, G., L. Catella y F. Oliva 2015. Nuevos datos de isótopos estables del C y N para el Sudeste de la Región Pampeana. Revista de Antropología del Museo de Entre Ríos 1:96-98.

Berón, M., L. Luna y R. Barberena 2009. Isotopic archaeology in the western pampas. International Journal of Osteoarchaeology 19:250-265.

Bonomo, M. 2005. Costeando las Llanuras. Arqueología del Litoral Marítimo Pampeano. Sociedad Argentina de Antropología, Buenos Aires.

Bonomo, M., C. Scabuzzo y C. Leon 2013. Cronología y dieta en la costa atlántica pampeana. Intersecciones en Antropología 14:5-18.
Bróndolo, M. y A. Zinger 1988. El espacio geográfico. En Historia del Sudoeste Bonaerense, editado por F. Weinberg y H. Silva, pp. 17-25. Plus Ultra, Buenos Aires.

Cabrera, A.L. 1976. Regiones Fitogeográficas Argentinas. ACME, Buenos Aires.

Flegenheimer, N., R. Guichón y C. Scabuzzo 2002. Restos óseos humanos en el sitio El Guanaco, partido de San Cayetano. En Del Mar a los Salitrales. Diez Mil Años de Historia Pampeana en el Umbral del Tercer Milenio, editado por D.L. Mazzanti, M.A. Berón y F.W. Oliva, pp. 121-126. UNMdelP, Mar del Plata.

Frère, M.M., D. Constenla, C. Bayón y M.I. González 2010. Estudios actualísticos sobre recursos silvestres mediante el empleo de análisis químico. En Mamül Mapu. Pasado y Presente desde la Arqueología Pampeana, editado por M. Berón, L. Luna, M. Bonomo, C. Montalvo, C. Aranda y M. Carrera Aizpitarte, pp. 65-75. Libros del Espinillo, Ayacucho.

Froehle, A.W., C.M. Kellner y M.J. Schoeninger 2012. Multivariate carbon and nitrogen stable isotope model for the reconstruction of prehistoric human diet. American Journal of Physical Anthropology 147:352-369.

Frontini, R. 2013. Aprovechamiento faunístico en entornos acuáticos del sudoeste bonaerense durante el Holoceno (6900-700 años AP). Relaciones de la Sociedad Argentina de Antropología XXXVIII:493-519.

Frontini, R. y C. Bayón 2015a. Use of marine resources (fauna and tool stones) in the southwest of Buenos Aires Province (Argentina) during the Middle and Late Holocene. En Zooarchaeology in the Neotropics: Environmental Diversity and Human Animal Interactions, editadopor M. Mondini, A.S. Muñoz y P.M. Fernández. Springer, New York. En prensa.

Frontini, R. y C. Bayón 2015b. Consumo de recursos animales de porte menor durante el Holoceno tardío en el sudoeste de la provincia de Buenos Aires (Argentina). Archaeofauna. International Journal of Archaeozoology 24:271-293.

Isla, F.I., L.C. Cortizo y H.A. Turno Orellano 2001. Dinámica y evolución de las barreras medanosas, Provincia de Buenos Aires, Argentina. Revista Brasileira de Geomorfología 2:73-83.

Katzenberg, A. 2008. Stable isotope analysis: A tool for studying past diet, demography, and life history. En Biological Anthropology of the Human Skeleton, editado por A. Katzenberg y S. Saunders, pp. 413-441. Willey Lis, New Jersey.

Loponte, D. y A. Acosta 2007. Horticultores amazónicos en el humedal del Paraná Inferior: los primeros datos isotópicos de la dieta. En Arqueología en las Pampas, editado por C. Bayón, 
I. González, N. Flegenheimer, A. Pupio y M. Frére, pp. 75-94. Sociedad Argentina de Antropología, Buenos Aires.

Madrid, P. y G. Barrientos 2000. La estructura del registro arqueológico del sitio Laguna Tres Reyes (Provincia de Buenos Aires): Nuevos datos para la interpretación del poblamiento humano del sudeste de la región pampeana a inicios del Holoceno Tardío. Relaciones de la Sociedad Argentina de Antropología XXV:179-206.

Martínez, G., G. Flensborg y P. Bayala 2012. Primeras evidencias de restos óseos humanos en el curso inferior del Río Colorado durante el Holoceno medio: sitio Cantera de Rodados Villalonga (Pdo. de Patagones, Pcia. de Buenos Aires). Cazadores Recolectores del Cono Sur 6:101-113.

Martínez, G., A. Zangrando y L. Prates 2009. Isotopic ecology and human paleodiets in the lower basin of the Colorado River, Buenos Aires province, Argentina. International Journal of Osteoarchaeology 19:281-296.

Matarrese, A. 2015. Tecnología Lítica entre los CazadoresRecolectores Pampeanos: Los Artefactos Formatizados por Picado, Abrasión y Modificados por Uso en el Área Interserrana Bonaerense. Tesis doctoral inédita. Facultad de Ciencias Naturales y Museo, Universidad Nacional de La Plata, La Plata.

Mazzia, N. y N. Flegenheimer 2015. Detailed fatty acids analysis on lithic tools, Cerro El Sombrero Cima, Argentina. Quaternary International 363:94-106.

Méndez, C., R. Barberena, O. Reyes y A. Nuevo Delaunay 2014. Isotopic ecology and human diets in the forest-steppe ecotone, Aisén Region, Central-Western Patagonia, Chile. International Journal of Osteoarchaeology 24:187-201.

Mendonça, O., A. Aguerre, M. Arrieta y L. Pera 2013. Investigaciones bioarqueológicas en la Laguna Chadilauquen, Embajador Martini, departamento Realicó, provincia de La Pampa. Segunda etapa. Revista del Museo de La Plata. Sección Antropología 13:137-152.

Murgo, A. y V. Aldazábal 2007. Análisis de isótopos estables de carbono y nitrógeno de los restos óseos humanos provenientes de sitios del área Pampa Deprimida centro oriental. En Arqueología Argentina en los Inicios de un Nuevo Siglo, editado por F. Oliva, N. de Grandis y J. Rodríguez, pp. 259-264. Universidad Nacional de Rosario, Rosario.

Pérez Amat, M. y D. Scheines de Tiverovsky 1978. Prehistoria. En Manual de Historia de Bahía Blanca, editado por F. Weimberg, pp. 33-48. Departamento de Ciencias Sociales, Universidad Nacional del Sur, Bahía Blanca.

Politis, G., M. Bonomo y L. Prates 2003. Territorio y movilidad entre la costa Atlántica y el interior de la región pampeana (Argentina). Estudos Ibero-Americanos XXIX(1):11-35.

Politis, G., C. Scabuzzo y R. Tykot 2009. An approach to prehispanic diets in the pampas during early/middle Holocene. International Journal of Osteoarchaeology 19:266-280.

Politis, G., G. Barrientos y T. Stafford 2011. Revisiting Ameghino: New ${ }^{14} \mathrm{C}$ dates from ancient human skeletons from the Argentine Pampas. En Pouplements etPréhistoireen Amérique, editado por D. Vialou, pp. 43-53.Éditions du Comité des travauxhistoriques et scientifiques, Paris.

Quattrocchio, M., E. Borromei, C. Deschamps, S. Grill y C. Zavala 2008. Landscape evolution and climate changes in the Late Pleistocene-Holocene, southern Pampa (Argentina): Evidence from palynology, mammals and sedimentology. Quaternary International 181:123-138.

Ringuelet, R. 1961. Rasgos fundamentales de la zoogeografía de la República Argentina. Physis 22:151-170.

Scabuzzo, C. 2013. Estudios bioarqueológicos del sitio Paso Mayor, sudoeste de la provincia de Buenos Aires. Revista del Museo de Antropología 6:49-62.

Scabuzzo, C. y M. González de Bonaveri 2007. Un acercamiento a la dieta de las poblaciones prehispánicas de la Depresión del Salado durante el Holoceno tardío. En Arqueología en las Pampas, editado por C. Bayón, N. Flegenheimer, M.I. González de Bonaveri y M. Frère, pp. 59-74. Sociedad Argentina de Antropología, Buenos Aires.

Tomassini, R., R. Frontini y C. Bayón 2014.Taphonomic analysis of a Lama guanicoe (Artiodactyla, Camelidae) assemblage from Late Holocene of Pampean region (Argentina). Palaios 29:570-577.

Vecchi, R., R. Frontini y C. Bayón 2013. Paso Vanoli: una instalación del Holoceno tardío en valles fluviales del sudoeste bonaerense. Revista del Museo de La Plata. Sección Antropología 13:77-93.

Zangrando, A.F., A. Tessone, A. Ugan y M.A. Gutiérrez 2014. Applications of stable isotope analysis in zooarchaeology: An introduction. International Journal of Osteoarchaeology 24:127-133. 
